\title{
SPECTRA OF INVARIANT UNIFORM \\ AND TRANSFORM ALGEBRAS
}

\author{
BY \\ I. GLICKSBERG ${ }^{1}$
}

\begin{abstract}
For $G$ a locally compact abelian group, any closed invariant proper subalgebra of $C_{0}(G)$ has analytic discs in its spectrum. Related results are given for $A(G)$ and $B(G)$.
\end{abstract}

For a compact group $G$ it is an easy consequence of Bishop's generalized Stone-Weierstrass theorem and some well-known facts on representations that a proper (doubly) invariant uniform algebra on $G$ cannot have $G$ as its spectrum. (Indeed, Haar measure of a nontrivial subgroup must be multiplicative.) The corresponding result for closed subalgebras of $C_{0}(G), G$ locally compact abelian, seemingly required more harmonic analysis (and, originally, the argument yielding Bishop's result); but more emerged, namely, that the spectra of (nonzero) proper closed invariant subalgebras of $C_{0}(G)$ contain analytic discs. This follows easily from the following more specific result.

THEOREM 1. Let $A$ be a separating translation invariant closed proper subalgebra of $C_{0}(G), G$ l.c.a. Then:

(1) there is a compact subgroup $H$ of $G$ whose normalized Haar measure $m$ is multiplicative on $A$, so $a \rightarrow m * a$ is a multiplicative projection of $A$ onto its subalgebra $A \cap C_{0}(G / H)$, while

(2) there is a nontrivial homomorphism $\sigma: \mathbf{R} \rightarrow G / H$ for which $\left[A \cap C_{0}(G / H)\right] \circ \sigma$ consists of boundary value functions (on $\mathbf{R}$ or $\mathbf{R} / \mathbf{Z}$ ) of continuous functions analytic on the closed half-plane or disc.

Alternatively, $A \cap C_{0}(G / H)$ consists of functions analytic with respect to the induced flow on $G / H$; in particular we have analyticity relative to a flow on $G$ when $G$ has no nontrivial compact subgroups. Of course one can exploit such analyticity; for example, if $f \in C_{0}\left(\mathbf{R}^{n}\right)$ has $f^{-1}(c)$ of positive Lebesgue measure for some $c \neq 0$ then $f$ and its translates generate $C_{0}\left(\mathbf{R}^{n}\right)$ as an algebra. Such corollaries are given in $\S 2$, with the first section devoted to the proof of our theorem.

After a revised version of this paper was essentially complete I found a result of $J$. L. Taylor [T1, Lemma 2] (extending, and using, an earlier result of Gleason and Rieffel [Ri, Theorem 6.4]), which is closely related to Theorem 2 below; at least two thirds of the proof of Theorem 1 is devoted to covering essentially the same ground

Received by the editors February 11, 1981 and, in revised form, May 10, 1982.

1980 Mathematics Subject Classification. Primary 46J10, 43A25.

${ }^{1}$ Work supported in part by the NSF. 
(by an apparently different route). Unfortunately Taylor errs in passing from the semicharacters obtained by Rieffel to the homomorphism he used, so that Taylor's result is incorrect as stated ${ }^{2}$ of course the correct reading of Taylor's proof is consequently made more difficult. A much stronger result was given later by Taylor [T3, Theorem 4.5.2.], but this requires more knowledge of measure algebras than one might expect from many readers interested in uniform algebras. As a consequence I have not altered this part of the original presentation, but all my results should properly be regarded as corollaries to the Gleason-Rieffel-Taylor result. (For the well-equipped reader the later Taylor result yields the neatest approach although all use the structure of l.c.a. groups.)

There remains the possibility of replacing $C_{0}(G)$ throughout by other algebras, in particular by the usual algebras of Fourier transforms, $A(G)$ and $B(G)$. It was only when prompted by this question from the referee that I noticed its simplicity for $A(G)$, and proceeding to $B(G)$, turned to Taylor's works. The results have been added in a final section.

1. Essentially as an aside, we shall start with the case of $G$ compact nonabelian because there the antisymmetric approach joins so easily with the harmonic analysis needed; the reader unfamiliar with or not interested in the latter can just as well skip to 1.1. (As the referee has pointed out, more detailed results have been given by Björk [B] and Gichev [Gi] for compact Lie groups.)

To begin, from invariance it is easy to see the maximal sets of antisymmetry for $A$ are all cosets of a compact normal subgroup $H$ (cf. [Gl.2, p. 127]) and as we shall see the Haar measure $m$ of $H$ is multiplicative on $A$. Indeed from Bishop's theorem [G] and the fact that $A$ is proper we know $H$ is nontrivial while $B=A \mid H$ is closed and proper. Of course it is an invariant algebra on $H$ and thus the span in $C(H)$ of all the entries in certain of $H$ 's unitary matricial representations. If $u_{i j}$ and $u_{k l}^{\prime}$ are two such entries, in $U, U^{\prime}$ say, and $U$ is not the trivial representation (so $m\left(u_{i j}\right)=0$ ) then $m$ annihilates $u_{i j} u_{k l}^{\prime}$ too since it is an entry in $U \otimes U^{\prime}$ which cannot contain the trivial representation unless $U^{\prime}$ is equivalent to $\bar{U}$ [N, p. 438], a contradiction to antisymmetry. Thus all trigonometric polynomials in $B$ with zero constant term form an ideal $I$ in the dense subalgebra $B_{p}$ of $B$ formed by its trigonometric polynomials, and $I$ is annihilated by $m$, as in $I^{-}$(an ideal in $B$ of course). Since any element of $B$ annihilated by $m$ can be approximated by elements of $I$ we clearly must have $B=\mathbf{C} \oplus I^{-}=\mathbf{C} \oplus\left(m^{-1}(0) \cap B\right)$, so $m$ provides an element of the spectrum of $A$ not in $G$, as desired.

1.1. In the analogous situation on an 1.c.a. group $G$ (with dual group $G^{\hat{}}=\Gamma$ ), we need not of course have any compact subgroups, and thus no potentially multiplicative (nontrivial) normalized Haar measure. Moreover, while antisymmetry was used

\footnotetext{
2 The correct assertion is that there is a continuous homomorphism from an open subgroup into $\mathbf{R}$ rather than from the full group, which is all Taylor subsequently uses. A simple counterexample to the result stated is the semigroup of nonnegative elements in a totally ordered discrete group for which, for each $x>0$ there is a $y>n x$, all $n \in \mathbf{Z}_{+}$.
} 
in this setting originally, and gave the direction our proof still follows, it has been replaced by a quite elementary argument.

Let $A^{-*}$ denote the $w^{*}$ closure of $A$ in $L^{\infty}(G)$. We shall view the dual group $\Gamma=\hat{G^{\wedge}}$ as a subset of $L^{\infty}(G)$, and accordingly write the operation as multiplication. Then

$$
S_{0}=\Gamma \cap A^{-*}
$$

is a subsemigroup of $\Gamma$ (since $A$ is a multiplicative subsemigroup on which the $w^{*}$ topology is separately continuous) which is closed since $\Gamma$ has the relative $w^{*}$ topology. Moreover, $S_{0}$ contains the spectrum ${ }^{3} \operatorname{sp}(a)$ of $a \in A$, and this fact will provide the basis for our proof; we shall show there is a homomorphism $\rho$ of an open subgroup $\Gamma_{1}$ of $\Gamma$ into $\mathbf{R}$ which is nonnegative (and nontrivial) on $S_{0} \cap \Gamma_{1}$; because of the Paley-Wiener theorem this yields analytic behavior for the subalgebra of elements constant modulo the subgroup $\Gamma_{1}^{\perp}$ of $G$, which essentially proves our theorem. Thus the main effort is to produce $\rho$.

To begin, note that our closed subsemigroup $S_{0}$ of $\Gamma$ is proper; if $\mu \neq 0$ is a measure orthogonal to our proper invariant algebra $A \subset C_{0}(G)$ then $\mu * f \neq 0$ for some $f$ in $L^{1}(G)$, and $\mu * f$, still orthogonal, is in fact orthogonal to $A^{-*}$. Since $A^{-*}$ cannot contain $\Gamma$ without containing its $w^{*}$ closed span, $L^{\infty}(G), S_{0}$ is proper.

Consequently, $L=S_{0} \cap S_{0}^{-1}$ is a closed proper subgroup; if the intersection is empty we let $L=\{1\}$. Now we reduce to the case in which $L$ is trivial: if $\pi_{0}: \Gamma \rightarrow \Gamma_{0}=\Gamma / L$ then $S=\pi_{0} S_{0}$ is a closed proper subsemigroup of $\Gamma_{0}$ with

$$
S \cap S^{-1} \subset\{1\} \text {. }
$$

Indeed, since $S_{0}$ is proper we have a $\gamma \in \Gamma \backslash S_{0}$ and for any open neighborhood $V$ of such a $\gamma$ missing $S_{0}$ we have $V L \cap S_{0}=\varnothing$ since otherwise $V \cap S_{0} \supset V \cap S_{0} L \neq \varnothing$. Thus $S$ is both closed and proper as asserted. Evidently to obtain a homomorphism $\rho$ of an open subgroup $\Gamma_{1}$ of $\Gamma$ into $\mathbf{R}$ which is $\geqslant 0$ and somewhere positive on $S_{0} \cap \Gamma$, it suffices to do the same for $S$ and $\Gamma_{0}$.

1.2. We should note that $S$ generates $\Gamma_{0}$ because $S_{0}$ generates $\Gamma$, i.e., $\left(S_{0} S_{0}^{-1}\right)^{-}=\Gamma$. Indeed, if the latter fails there is an $x$ in $G$ orthogonal to $S_{0} S_{0}^{-1}$ and not the identity of $G$. So for any $f$ in $L^{1}(G), f_{x}-f$ annihilates $\left(S_{0} S_{0}^{-1}\right)^{-} \subset L^{\infty}(G)$, hence annihilates any element of the $w^{*}$ closed span of $\left(S_{0} S_{0}^{-1}\right)^{-}$. But the latter contains $A$ since, being a closed subgroup, $\left(S_{0} S_{0}^{-1}\right)^{-}$is a set of synthesis by Reiter's theorem $[\mathbf{H}, \mathbf{R}]$. Thus $f_{x} * a=f * a=(f * a)_{x}$ for all $f$ in $L^{1}(G)$ and $a \in A$, so a dense subalgebra of $A$ cannot separate $x$ and the identity, and $A$ cannot.

1.3. We now proceed to show there is an open subgroup $\Gamma_{1}$ of $\Gamma_{0}$ and homomorphism $\rho: \Gamma_{1} \rightarrow \mathbf{R}$ which is nonnegative on $\Gamma_{1} \cap S$ and somewhere positive. To do so we shall use the fact that $\Gamma_{0}=\Gamma_{*} \times \mathbf{R}^{n}$ where $\Gamma_{*}$ has a compact open subgroup $K$ and $n \geqslant 0[\mathbf{R u}, \mathbf{W e}]$.

\footnotetext{
${ }^{3} \mathrm{sp}(\mathrm{a})=\bigcap\left\{\hat{\mu}^{-1}(0): \mu * a=0, \mu \in L^{1}\left(G_{0}\right)\right\}$ (where $L^{1}$ can be replaced by $M$ ); dually $\operatorname{sp}(a)=\Gamma \cap$ $\operatorname{span}\left\{a_{x}: x \in G_{0}\right\}^{-*}$. Thus $S=A^{-*} \cap \Gamma \supset\left(\bigcup_{a \in A} \operatorname{sp}(a)\right)$, but in fact one has equality: for $\gamma$ not in the second set one has $\mu \in L^{1}$ with $\hat{\mu} \equiv 0$ near the second so $\mu * a=0, a \in A$, by spectral synthesis for open sets, whence $\gamma$ is separated from $A^{-*}$.
} 
Let

$$
\pi: \Gamma_{0}=\Gamma_{*} \times \mathbf{R}^{n} \rightarrow \Gamma_{*} / K
$$

denote the natural homomorphism and $S_{1}=\pi S$; since $\Gamma^{\prime}=\Gamma_{*} / K$ is discrete and $S S^{-1}$ dense in $\Gamma_{0}$, evidently $S_{1} S_{1}^{-1}=\Gamma^{\prime}$. We shall consider the two possible cases:

$$
\begin{aligned}
& S_{1} \neq \Gamma^{\prime} \quad\left(\text { so } S_{1} \cap S_{1}^{-1} \neq \Gamma^{\prime}\right), \\
& S_{1}=\Gamma^{\prime} \quad\left(\text { so } S_{1}^{-1}=\Gamma^{\prime} \text { as well }\right) .
\end{aligned}
$$

1.4. In case (i) let $S_{1}^{\prime}$ denote the semigroup $\{1\} \cup S_{1}$, so that $L^{\prime}=S_{1}^{\prime} \cap S_{1}^{\prime-1}$ is a nonempty subgroup of $\Gamma^{\prime}$, and proper: for at most the identity has been added to the intersection, and $S_{1} \cap S_{1}^{-1}=\Gamma^{\prime} \backslash\{1\}$ implies $1=\gamma \gamma^{-1} \in S_{1} \cap S_{1}^{-1}$ for any $\gamma \neq 1$ (such a $\gamma$ exists unless $\Gamma^{\prime}$ is a singleton, and then only case (ii) can occur since $\left.S_{1} \neq \varnothing\right)$. Now let $\pi_{1}: \Gamma^{\prime} \rightarrow \Gamma^{\prime} / L^{\prime}, S_{2}=\pi_{1} S_{1}^{\prime}$. Then $S_{2} \cap S_{2}^{-1}=\{1\}$ since $\gamma L^{\prime}=$ $\gamma_{1}^{-1} L^{\prime}, \gamma, \gamma_{1} \in \Gamma^{\prime}$, implies $\gamma \gamma_{1} \in L^{\prime}$, so $\gamma \gamma_{1}=\gamma_{2}^{-1}, \gamma_{2} \in S_{1}$, whence $\gamma=\left(\gamma_{1} \gamma_{2}\right)^{-1} \in$ $S_{1} \cap S_{1}^{-1}=L^{\prime}$. Among the subsemigroups $S^{\prime}$ of $\Gamma^{\prime}$ containing $S_{2}$ and satisfying

$$
S^{\prime} \cap\left(S^{\prime}\right)^{-1}=\{1\}
$$

we have a maximal one, $S_{3}$ say. This has the property that every $\gamma \in S_{3}, \gamma \neq 1$, is of infinite order because of (4) since $\gamma^{n}=1$ with $n>1$ least implies $\gamma^{n-1}=\gamma^{-1} \in S_{3}$ $\cap S_{3}^{-1}=\{1\}$. In addition, by maximality $\gamma \in \Gamma^{\prime} \backslash\left(S_{3} \cup S_{3}^{-1}\right)$ is of finite order; for if not, either no power of $\gamma$ lies in $S_{3} \cup S_{3}^{-1}$ and we can enlarge $S_{3}$ to the subsemigroup generated by $\gamma$ and $S_{3}$ and preserve (4) (since $\gamma^{k} s=\gamma^{-l} s_{1}^{-1}$ for $k, l>0, s, s_{1} \in S_{3}$ implies $\left.\gamma^{k+l}=\left(s s_{1}\right)^{-1}\right)$, or some power lies in $S_{3}$ or $S_{3}^{-1}$. But $\gamma^{k} \in S_{3}$ (resp. $S_{3}^{-1}$ ) allows us also to adjoin $\gamma$ to $S_{3}$ (resp. $S_{3}^{-1}$ ); for $\gamma^{j} s=\gamma^{-l} s_{1}^{-1}$ implies $\left(\gamma^{j+l}\right)^{k}=\left(s s_{1}\right)^{-k} \in S_{3} \cap S_{3}^{-1}$ so $=1$.

Thus every element of $S_{3} \cup S_{3}^{-1}$ (save 1) is of infinite order while every element of the complement is of finite order. So if $\Gamma^{\prime \prime}$ denotes the subgroup of elements of finite order the image $S_{4}$ of $S_{3}$ in $\Gamma^{\prime} / \Gamma^{\prime \prime}$ has the property that $S_{4} \cap S_{4}^{-1}=\{1\}$ and $S_{4} \cup S_{4}^{-1}=\Gamma^{\prime} / \Gamma^{\prime \prime}$. Hence $S_{4}$ provides a total ordering of $\Gamma^{\prime} / \Gamma^{\prime \prime}$, making $\Gamma^{\prime} / \Gamma^{\prime \prime}$ an ordered abelian group. Selecting any element $\gamma_{0} \neq 1$ of the image $\tilde{S}$ of $S$ in $S_{4}$ the subset of $\Gamma^{\prime} / \Gamma^{\prime \prime}$ bounded by $\gamma_{0}$ in that order,

$$
\Gamma^{\prime \prime \prime}=\bigcup_{n}\left(\gamma_{0}^{-n} S_{4} \cap \gamma_{0}^{n} S_{4}^{-1}\right),
$$

forms a subgroup of $\Gamma^{\prime} / \Gamma^{\prime \prime}$ whose complement meets $S_{4}$, or better, the homomorphic image $\tilde{S}$ of $S$ itself, in an ideal of that semigroup, as is easily seen (elements which are infinitely large relative to $\gamma_{0}$ in the ordering multiply elements of $S_{4}$ to yield infinitely large elements). By dividing out the subgroup of infinitely small elements in $\Gamma^{\prime \prime}$ we obtain an archimedian ordered group so we have a nonnegative homomorphism of $\Gamma^{\prime \prime \prime}$ into $\mathbf{R}$ sending $\gamma_{0}$ into $1 \in \mathbf{R}$ say; the inverse of $\Gamma^{\prime \prime \prime}$ back in $\Gamma_{0}$ itself yields the desired open subgroup $\Gamma_{1}$ on which we have a homomorphism $\rho$ into $\mathbf{R}$ sending $S \cap \Gamma_{1}$ into $\mathbf{R}_{+}$, with some positive value there. Equivalently, pulling back to $\Gamma$ from $\Gamma_{0}=\pi_{0} \Gamma=\Gamma / L$ we have a homomorphism (still called $\rho$ ) of an open subgroup $\pi_{0}^{-1} \Gamma_{1}$ of $\Gamma$ into $\mathbf{R}$ which is nonnegative on $S_{0} \cap \pi_{0}^{-1} \Gamma_{1}$ and somewhere positive. Finally, if $m$ denotes the normalized Haar measure of the 
compact subgroup $H=\left(\pi_{0}^{-1} \Gamma_{1}\right)$ of $G$ (whose dual is the discrete group $\Gamma / \pi_{0}^{-1} \Gamma_{1}$ ), the fact that $\tilde{S} \backslash \Gamma^{\prime \prime \prime}$ is an ideal in $\tilde{S}$, hence that $S \backslash \Gamma_{1}$ is one in $S$, and $S_{0} \backslash \pi_{0}^{-1} \Gamma_{1}$ is one in $S_{0}$, shows $m$ is multiplicative on $A$ as we next note.

1.5. Indeed the invariant algebra $(A \mid H)^{-}$on the compact group $H$ is the uniform closure of the combined spectra of the elements $a \mid H$ of $A \mid H$, while $\operatorname{sp}(a \mid H) \subset$ $\pi(\operatorname{sp}(a))^{-}$where $\pi: \Gamma \rightarrow \Gamma /\left(\pi_{0}^{-1} \Gamma_{1}\right)^{\perp}=H^{\wedge}$ is the natural map [R, p. 143]. Because $H^{\hat{H}}$ is discrete,

$$
\operatorname{sp}(a \mid H) \subset \pi \operatorname{sp}(a) \subset \pi S_{0},
$$

and $A \mid H \subset$ span $\pi S_{0}$. Since, as functions on $H$,

$$
\pi S_{0}=S_{0}\left|H=\left(S_{0} \cap \pi^{-1} \Gamma_{1}\right)\right| H \cup\left(S_{0} \backslash \pi_{0}^{-1} \Gamma_{1}\right) \mid H
$$

while $m(\gamma)=m(1)=1$ on the first set in the union and $\equiv 0$ on the ideal formed by the second set, $m$ must be multiplicative on their uniform span, hence on $A \mid H$, and A.

1.6. We can now turn to case (ii), where

$$
\pi: \Gamma_{0}=\Gamma_{*} \times \mathbf{R}^{n} \rightarrow \Gamma_{*} / K=\Gamma^{\prime}
$$

maps $S$ and $S^{-1}$ onto $\Gamma^{\prime}$. Recalling (2), that $S \cap S^{-1} \subset\{1\}$, we can now note that since a closed subsemigroup of a compact abelian group is a group [W, Gl.1], this implies $(K \times\{0\}) \cap S$ contains at most the identity. As a first consequence of this if $n=0, \Gamma^{\prime}$ is nontrivial (since $S$ cannot reduce to the identity). Indeed if $n=0$ (so $\Gamma=\Gamma_{*}$ ) and if $\gamma \in \Gamma \backslash K$ then there is a $k \in K$ for which $\gamma k \in S$ since $\pi$ maps $S$ onto $\Gamma^{\prime}$; similarly there is a $k^{\prime} \in K$ with $\gamma^{-1} k^{\prime} \in S$. But then $k k^{\prime}=\gamma k \gamma^{-1} k^{\prime} \in K \cap$ $S \subset\{1\}$ so $k^{\prime}=k^{-1}$ and thus $\gamma^{-1} k^{-1} \in S$ so $\gamma k \in S \cap S^{-1} \subset\{1\}$ despite $\gamma \notin K$. So $n \geqslant 1$.

Next, if

$$
\pi_{2}: \Gamma_{*} \times \mathbf{R}^{n} \rightarrow \mathbf{R}^{n} \quad \text { and } \quad S_{*}=\left(K \times \mathbf{R}^{n}\right) \cap S
$$

we have $\left(\pi_{2} S_{*}\right)^{-} \cap\left(\pi_{2} S_{*}^{-1}\right)^{-}=\left(\pi_{2} S_{*}\right)^{-} \cap\left(\left(\pi_{2} S_{*}\right)^{-}\right)^{-1}$ containing at most the identity of $\mathbf{R}^{n}$; for $r$ therein implies we have $\left(k_{j}, r_{j}\right)$ and $\left(k_{j}^{\prime}, r_{j}^{\prime}\right)$ in $S^{*}$ with $r_{j} \rightarrow r$, $r_{j}^{\prime} \rightarrow-r$. Passing to subnets we can assume $k_{j} \rightarrow k, k_{j}^{\prime} \rightarrow k^{\prime}$ in $K$, so that $\left(k k^{\prime}, 0\right) \in$ $S_{*}=\left(K \times \mathbf{R}^{n}\right) \cap S$, hence lies in $(K \times\{0\}) \cap S \subset\{1\}$. Thus $k^{\prime}=k^{-1}$, so $(k, r)$ and $\left(k^{-1},-r\right)$ both lie in $S_{*} \subset S$, so coincide with the identity by (3), and $r=0$ as asserted.

Actually, $S_{*} \neq \varnothing$ since $S$ maps onto $\Gamma^{\prime}=\Gamma_{*} / K$ so $S_{* *}=\left(\pi_{2} S_{*}\right)^{-}$is a nonempty closed subsemigroup of $\mathbf{R}^{n}$ with $S_{* *} \cap\left(-S_{* *}\right) \subset\{0\}$. Moreover, the set of positive dilates of $S_{* *}, \mathbf{R}_{+} S_{* *}$, meets its negative at most in the identity; for $c, c^{\prime}>0, s, s^{\prime}$ in $S_{* *}, c s=-c^{\prime} s^{\prime}$, or $s=-\left(c^{\prime} / c\right) s^{\prime}$, cannot hold if $c^{\prime} / c$ is rational since then $k s=-l s^{\prime}$ for positive integers $k$, $l$, whence both lie in $S_{* *} \cap\left(-S_{* *}\right)$, hence $=0$; and if $c^{\prime} / c$ is irrational $S_{* *}$ contains $\left\{k s-l\left(c^{\prime} / c\right) s: k, l \in \mathbf{Z}_{+}\right\}$which is dense in the line through 0 and $s$, so $s \neq 0$ implies $S_{* *}$, and so $S_{* *} \cap\left(-S_{* *}\right)$, contains a line through 0 . Thus

$$
\left(-\mathbf{R}_{+} S_{* *}\right) \cap\left(\mathbf{R}_{+} S_{* *}\right) \subset\{0\} .
$$


Now suppose $S_{* *}$ contains a maximal independent set of $k \leqslant n$ elements. Then in their vector span $V$ over $\mathbf{R}, \mathbf{R}_{+} S_{* *}$ has interior, so (6) implies $\left(\mathbf{R}_{+} S_{* *}\right)^{-}$misses the $k$-dimensional interior of its negative, and thus $\left(\mathbf{R}_{+} S_{* *}\right)^{-}$is a proper closed cone in $V$, hence in $\mathbf{R}^{n}$. Consequently we have a nontrivial linear functional $\psi$ on $\mathbf{R}^{n}$ which is nonnegative on $S_{* *}=\pi_{2}\left(\left(K \times \mathbf{R}^{n}\right) \cap S\right)^{-}$and indeed positive at some point of $\pi_{2} S$.

For the moment let $\Gamma^{*}=K \times \mathbf{R}^{n}$. Then for $\gamma, \gamma^{\prime} \in \Gamma_{0}$ trivially

$$
\left(S \cap \gamma \Gamma^{*}\right)\left(S \cap \gamma^{\prime} \Gamma^{*}\right) \subset S \cap \gamma \gamma^{\prime} \Gamma^{*},
$$

so if we set

$$
h(\gamma)=\inf \left\{\psi\left(\pi_{2}\left(\gamma^{\prime}\right)\right): \gamma^{\prime} \in S \cap\left(\gamma \Gamma^{*}\right)\right\}
$$

then $h(1) \geqslant 0$ and (7) implies (since each set is nonvoid) that

$$
h(\gamma)+h\left(\gamma^{\prime}\right) \geqslant h\left(\gamma \gamma^{\prime}\right) \text {. }
$$

In particular $h(\gamma)+h\left(\gamma^{-1}\right) \geqslant h(1) \geqslant 0$, and $h(\gamma)$ is always finite, while $h$ is constant on cosets mod $\Gamma^{*}$.

Now consider

$$
\Sigma=\left\{(\gamma, t) \in \Gamma_{*} \times \mathbf{R}: t>h(\gamma)\right\} .
$$

From (8) we conclude $\Sigma$ is a subsemigroup of $\Gamma_{*} \times \mathbf{R}$; moreover $\Sigma \cap \Sigma^{-1}=\varnothing$ since $(\gamma, t) \in \Sigma \cap \Sigma^{-1}$ says $t>h(\gamma)$ and $-t>h\left(\gamma^{-1}\right)$, so $0>h(\gamma)+h\left(\gamma^{-1}\right) \geqslant$ $h(1) \geqslant 0$. Now among the subsemigroups $\Sigma^{\prime}$ of $\Gamma_{*} \times \mathbf{R}$ containing $\Sigma$ with $\Sigma^{\prime} \cap\left(\Sigma^{\prime}\right)^{-1}$ containing at most the identity, we have a maximal subsemigroup $\Sigma^{*}$, and exactly as in case (i) (cf. 1.4), we conclude that any element of $\Sigma^{*}$, other than the identity, has infinite order, while $\left(\Gamma_{*} \times \mathbf{R}\right) \backslash\left(\Sigma^{*} \cup \Sigma^{*-1}\right)$ consists of elements of finite order (which necessarily have second coordinate zero). As before, if $\Gamma^{* *}=K \times \mathbf{R}$

$$
\left(\Sigma^{*} \cap \gamma \Gamma^{* *}\right) \cdot\left(\Sigma^{*} \cap \gamma^{\prime} \Gamma^{* *}\right) \subset \Sigma^{*} \cap \gamma \gamma^{\prime} \Gamma^{* *}, \quad \gamma, \gamma^{\prime} \in \Gamma^{*} \times \mathbf{R},
$$

which shows that with $H(\gamma)=\inf \left\{\pi_{2} \gamma^{\prime}: \gamma^{\prime} \in \Sigma^{*} \cap \gamma \Gamma^{* *}\right\}$ we have

$$
H\left(\gamma \gamma^{\prime}\right) \leqslant H(\gamma)+H\left(\gamma^{\prime}\right)
$$

again. From this it follows that $H(1) \geqslant 0$ if it is finite; but $\Sigma \supset \Gamma_{*} \times(h(1), \infty)$, or $\Gamma_{*} \times(-\infty,-h(1)) \subset \Sigma^{-1} \subset \Sigma^{*-1}$, yields finiteness of $H(1)$, hence of $H(\gamma)$ since $H(\gamma)+H\left(\gamma^{-1}\right) \geqslant H(1)$. But now $H$ is a homomorphism; for $\varepsilon>0$, writing $\gamma=$ $(\eta, t) \in \Gamma_{*} \times \mathbf{R}$ and $H(\eta)$ for $H(\gamma)$ (which is independent of $\left.t\right)$ we have $(\eta, H(\eta)-$ $\varepsilon) \in\left(\Sigma^{*}\right)^{-1}$ provided $H(\eta)-\varepsilon \neq 0$, so $-H(\eta)+\varepsilon \geqslant H\left(\eta^{-1}\right)$, whence $-H(\eta) \geqslant$ $H\left(\eta^{-1}\right)$ for all $\eta \in \Gamma_{*}$. But then $0 \leqslant H(1) \leqslant H(\eta)+H\left(\eta^{-1}\right) \leqslant 0$ follows so $H\left(\eta^{-1}\right)$ $=-H(\eta)$, and thus

$$
H(\eta)=H\left(\eta \eta_{1} \eta_{1}^{-1}\right) \leqslant H\left(\eta \eta_{1}\right)+H\left(\eta_{1}^{-1}\right)=H\left(\eta \eta_{1}\right)-H\left(\eta_{1}\right)
$$

which combines with (9) (written in our abused notation as $H\left(\eta \eta_{1}\right) \leqslant H(\eta)+H\left(\eta_{1}\right)$ ) to yield our assertion.

Now we simply have to note that $h \geqslant H$ automatically, so that (recalling the definition of $h) \psi\left(\pi_{2}(\gamma)\right) \geqslant H(\gamma), \gamma \in S$; thus $\psi \circ \pi_{2}-H$ provides a homomorphism of $\Gamma_{0}$ into $\mathbf{R}$, nonnegative on $S$ and somewhere positive since $\psi\left(\gamma_{0}\right)>0$ for 
some $\gamma_{0}$ in $\left(K \times \mathbf{R}^{n}\right) \cap S$ while $H\left(\gamma_{0}\right)=H(1)=0$. (Since $H^{-1}\left(\mathbf{R}_{+}\right)$has interior, it is easy to see $H$ is continuous; for discontinuity at $\gamma_{0}=1$ would imply $\varlimsup_{\gamma \rightarrow \gamma_{0}} H(\gamma)$ $=+\infty$ and $\lim _{\gamma \rightarrow \gamma_{0}} H(\gamma)=-\infty$, and by translation this would hold at any $\gamma_{0}$.)

1.7. We have now obtained in each case a compact subgroup $H$ (trivial in case (ii)) whose normalized Haar measure $m$ is multiplicative on $A$ along with a nontrivial homomorphism $\rho:(G / H) \hat{)} \rightarrow \mathbf{R}$ which is $\geqslant 0$ and somewhere $>0$ on $S_{0}$. Dually we have a map $\hat{\rho}: \mathbf{R} \rightarrow G / H$ which provides our flow on $G / H$ by translation. Evidently $a \rightarrow m * a$ maps $A$ multiplicatively onto its subalgebra $m * A$ of functions constant on cosets $\bmod H$, and it remains to see each such $a$ has $a \circ \hat{\rho}$, the boundary value function of a function analytic in a half-plane or disc.

For this it suffices to see that ${ }^{4} \operatorname{sp}(a \circ \hat{\rho}) \subset \mathbf{R}_{+}$. Now

$$
\operatorname{sp}(a \circ \hat{\rho})=\bigcap\left\{\hat{\mu}^{-1}(0): \mu \in M(R), \mu *(a \circ \hat{\rho})=0\right\},
$$

while $\mu *(a \circ \hat{\rho})=0$ iff $\hat{\rho}^{*} \mu * a=0$, where $\hat{\rho}^{*} \mu$ is the image of $\mu$ on $G / H$. Moreover $\left(\hat{\boldsymbol{\rho}}^{*} \mu\right) \hat{(\gamma)}=\hat{\mu}(\gamma \circ \hat{\boldsymbol{\rho}})=\hat{\mu} \circ \rho(\gamma)$. Suppose $t<0$. Then we have $\mu \in L^{1}(\mathbf{R}) \subset M(\mathbf{R})$ with $\hat{\mu}(t)=1, \hat{\mu} \equiv 0$ near $\mathbf{R}_{+}$, so $\left(\hat{\rho}^{*} \mu\right) \hat{\mu}=\hat{\mu} \circ \equiv 0$ near $\rho^{-1}\left(\mathbf{R}_{+}\right) \supset S \supset \operatorname{sp}(a)$. Thus $\hat{\rho}^{*} \mu * a=0$ by spectral synthesis for open sets, so $\mu *(a \circ \hat{\rho})=0$. Since $\hat{\mu}(t)=1, t \notin \operatorname{sp}(a \circ \hat{\rho})$, and our proof is complete.

The two cases we were forced to consider in 1.3 correspond exactly to the two possibilities for a representation $\hat{\rho}$ of $\mathbf{R}$ in an l.c.a. group [We, p. 96]; either (i) $\hat{\rho}(\mathbf{R})^{-}$ is a compact subgroup or (ii) $\hat{\rho}$ is an isomorphism. (Case (i) leads to a $\hat{\rho}$ which factors through a discrete group, so $\hat{\rho}(\mathbf{R})^{-}=H$ is compact (cf. 1.4), and in case (ii) this cannot be the case since we have a line in $\Gamma$ mapping onto $\mathbf{R}$ under $\hat{\rho}$ and since $\mathbf{R} \rightarrow \Gamma \rightarrow \mathbf{R}$ is topological so is the dual.) We shall make use of this dichotomy later.

Finally it is easy to see the above proof applies equally well to a strictly closed subalgebra of $C(G)$ if we simply replace closure in $C_{0}$ everywhere by strict closure in C. Thus

THEOREM 1'. Let $A$ be a separating translation invariant strictly closed proper subalgebra of $C(G), G$ l.c.a. Then the conclusions of Theorem 1 apply (with $C$ in place of $C_{0}$ ).

1.8. Clearly a large portion of our proof yields an assertion about certain closed subsemigroups of 1.c.a. groups $\Gamma$. Call $E \subset \Gamma$ a half of $\Gamma$ if there is a nontrivial homomorphism $\rho$ of $\Gamma$ into an 1.c.a. group $\Pi$ which is totally ordered by its closed subsemigroup $\Pi_{+}$of nonnegative elements, while $E=\rho^{-1} \Pi_{+}$. As a direct corollary of our proof we have

THEOREM 2. A closed proper generating subsemigroup $S$ of an l.c.a. group $\Gamma$ is contained in a half of $\Gamma$.

\footnotetext{
${ }^{4}$ This is a well-known consequence of the Paley-Wiener theorem; indeed if $\operatorname{sp}(\varphi) \subset[0, \infty)$ and $\left\{f_{n}\right\}$ is an approximate identity with $f_{n} \in L^{1} \cap L^{2}(\mathbf{R})$ and having support in $(-1 / n, 1 / n)$ then $\hat{f}_{n} \varphi \in L^{2} \cap L^{\infty}(\mathbf{R})$ has $\operatorname{sp}(\hat{f} \varphi) \subset[-1 / n, \infty)$ so that $\varphi_{n}=\exp \left(\right.$ in $\left.^{-1} \cdot\right) \hat{f}_{n} \varphi \in H^{2} \cap L^{\infty}=H^{\infty}$ by Paley-Wiener, with $L^{\infty}$. norm $\leqslant\|\varphi\|_{\infty}$. Since $\hat{f}_{n} \rightarrow 1$ uniformly on compacta it is easy to conclude $\varphi$ is the boundary value function of any normal family limit of $\left\{\varphi_{n}\right\}$ on $\mathcal{H}_{+}$.
} 
Actually $\Pi$ is $\mathbf{R}$ or discrete, corresponding to our two cases. Note that if we let $A$ be the strictly closed span of $S$ then $A$ is an invariant algebra, and proper in $C(G)$; indeed since $\gamma \in \Gamma \backslash S$ can be separated from $S$ by a measure in $L^{1}(G), S=\Gamma \cap A$. Thus any closed proper subsemigroup $S$ of $\Gamma$ arises as $A^{-} \cap \Gamma$ for a subalgebra of $C(G)$ under the strict topology. This is certainly not the case for subalgebras of $C_{0}(G)$ (where $A^{-}$is to be taken as the $w^{*}$ closure in $L^{\infty}(G)$ ): $S$ must be locally an $M$ set, i.e., for any closed neighborhood $N$ of each $\gamma$ in $S$ there must be $\varphi \in C_{0}, \varphi \neq 0$, with $\operatorname{sp}(\varphi) \subset N \cap S$. (Indeed some $a \in A$ has $\operatorname{sp}(a) \cap N^{0} \neq \varnothing$ (cf. footnote 3) so $\varphi=f * a$ will do for some $f$ in $L^{1}$.) Conversely such an $S$ will be $A^{-*} \cap \Gamma$ for the subalgebra $A$ of $C_{0}(G)$ generated by all $\varphi$ in $C_{0}$ with $\operatorname{sp}(\varphi) \subset S$.

We should note that, at least in the strict setting, $A$ need not be determined by $S$ when $G$ is noncompact precisely because of the failure of spectral synthesis. ${ }^{5}$

2. We can now easily prove

COROLlaRY 1. If $A$ is a (strictly) closed proper invariant subalgebra of $C_{0}(G)$ (resp. $C(G))$ then the spectrum of $A$ contains analytic discs.

In fact the discs will appear as an extension to the disc $D$, or upper half-plane $\mathcal{H}_{+}$, of $\hat{\rho}$, so our analytic disc will be "bounded" by a line or circle in our group or a quotient thereof.

Suppose for the moment $A$ separates $G$ so our theorems apply. Then either $\hat{\rho}$ is not $1-1$ and $a \circ \hat{\rho}$ is the restriction to $\partial D$ of a function in the disc algebra $A(D)$ for $a \in A_{1}=m * A$, or $a \circ \hat{\rho} \in H^{\infty}\left(\mathcal{H}_{+}\right)$. In either case if $\lambda_{z}$ is the usual representing measure on $\partial D$ or $\partial \mathcal{H}_{+}$we know the analytic extension of $a \circ \hat{\rho}$ is given by

$$
z \rightarrow \lambda_{z}(a \circ \hat{\rho})=\int a(\hat{\rho}(t)) \lambda_{z}(d t)
$$

since $\lambda_{z}$ is appropriately multiplicative the map into $A(D)$ or $H^{\infty}\left(\mathcal{H}_{+}\right)$is also, and $a \rightarrow \lambda_{z}(a \circ \hat{\rho})$ is a multiplicative linear functional on $A_{1}$. To obtain the corresponding element of the spectrum of $A$ we need only apply our multiplicative projection first:

$$
\begin{aligned}
a & \rightarrow \int m * a(\hat{\rho}(t)) \lambda_{z}(d t)=\int m * a(x) \hat{\rho}^{*} \lambda_{z}(d x) \\
& =\iint a(x+y) m(d y) \hat{\rho}^{*} \lambda_{z}(d x)=m * \hat{\rho}^{*} \lambda_{z}(a)
\end{aligned}
$$

\footnotetext{
${ }^{5}$ If $K$ is a compact set of nonsynthesis and $x \in G$ has $n x \rightarrow \infty$ then the closed subsemigroup $S$ generated by $N x+K$ (for some large $N$ ) will meet a neighborhood $V$ of $N x+K$ in just that set. Let $\mu$ be a measure with support precisely $N x+K, A$ the strictly closed invariant subalgebra of $C(G)$ generated by $\hat{\mu}$ (so $A \cap \Gamma=S$ ) and let $\varphi \in C(G)$ have $\operatorname{sp}(\varphi) \subset N x+K$ and not lie in the strictly closed span of $N x+K$ in $C(G)$ (which exists by failure of synthesis). Then $\varphi \notin A$ since if $a_{\delta} \rightarrow \varphi$ strictly and $f \in L^{1}(G)$ has $\hat{f} \equiv 1$ near $N x+K$ but $\equiv 0$ off $V$ then $f * a_{\delta} \rightarrow f * \varphi=\varphi$ strictly, while $f * a_{\delta}$ lies in the strictly closed span of $N x+K$ since $f$ annihilates span $(S \backslash(N x+K))$. Consequently, the strictly closed invariant algebra $B$ generated by all $\psi$ in $C(G)$ with spectra in $N x+K$ contains $A$ properly and has $B \cap \Gamma=S$.
} 
provides our element of the spectrum with $m * \hat{\rho}^{*} \lambda_{z}$ our representing measure (familiar from [AS]). Now $z \rightarrow m * \hat{\rho} * \lambda_{z}$ provides our analytic disc once we see this map is $1-1$; since $a \circ \hat{\rho}$ nonconstant implies (10) is nonconstant we of course have the map 1-1 on subdiscs of $D$ or $\mathcal{K}_{+}$, but in fact we have global 1-1-ness. This is easier to see in the disc case: if the rotation invariant algebra $A \circ \hat{\rho}$ does not separate $z_{1} \neq z_{2}$ in $D$ and $\left|z_{1}\right|=\left|z_{2}\right|=r$ then $t=z_{1} / z_{2} \in \partial D$ is a period of our functions on $|z|=r$ so $f \in A \circ \hat{\rho}$ implies $f(z)-f(t z) \equiv 0$ on $|z|=r$, so on $D$, and $A \circ \hat{\rho}$ cannot separate $\partial D$. Again if $r_{1}=\left|z_{1}\right|<\left|z_{2}\right|=r_{2}$ then invariance again implies $f \in A \circ \hat{\rho}$ has the same range on $|z|=r_{1}$ as on $|z|=r_{2}$, so any nonconstant $f$ attains its maximum modulus over $|z| \leqslant r_{2}$ at an interior point.

In the nondisc case, if $z_{1} \neq z_{2}$ in $\mathcal{H}_{+}$are not separated and $\operatorname{Im} z_{1}=\operatorname{Im} z_{2}$ then $z_{1}-z_{2} \in \mathbf{R}$ is a period of $f \in A \circ \hat{\boldsymbol{\rho}}$ on the horizontal line $\operatorname{Im} z=\operatorname{Im} z_{1}$ so a period of $f$ as before, and $A$ cannot separate $\hat{\rho}(\mathbf{R})$. If $0 \leqslant \operatorname{Im} z_{1}<\operatorname{Im} z_{2}$ we have two subcases corresponding to the possibilities for $\hat{\rho}$, cited earlier [We, p. 96]; either $\hat{\rho}$ is an isomorphism or $\hat{\boldsymbol{\rho}}(\mathbf{R})^{-}$is a compact subgroup. In the first subcase $f \in A \circ \hat{\boldsymbol{\rho}} \in$ $C_{0}(\mathbf{R})$ so its analytic extension to $\mathcal{H}_{+}$vanishes at $\infty$ by a well-known Phragmén-Lindelöf theorem [Ti, 5.63], and $f\left(\cdot+z_{1}\right)$ thus assumes its maximum modulus at some $z$ in $\mathbf{R}$, hence at some $z$ in $\mathbf{R}+\left(z_{2}-z_{1}\right)$, interior to $\mathcal{H}_{+}$. In the second subcase $A \circ \hat{\rho}$ consists of almost periodic functions. If $0=\operatorname{Im} z_{1}$, so we can assume $z_{1}=0$, we can choose any nonconstant $f \in A \circ \hat{\rho}$ of norm 1 and $x_{n}$ in $\mathbf{R}$ with $\left|f\left(-x_{n}\right)\right| \rightarrow 1$; then $\left\{f_{x_{n}}\right\}$ has a uniform cluster point $g$ in $A \circ \hat{\rho}$ with $\|g\|=1=|g(0)|=\left|g\left(z_{1}\right)\right|$ and since $g$ cannot separate $z_{1}=0$ and $z_{2}, g$ assumes its maximum modulus at an interior point so is constant. But that is impossible since any $g$ in the closed orbit $\left\{f_{x}: x \in \mathbf{R}\right\}^{-}$of $f$ has precisely the same orbit as $f$. Finally, in case $0<\operatorname{Im} z_{1}$ we have only to replace $f$ by $f_{1}=\lambda_{z_{1}} * f \in A \circ \hat{\rho}$; then $f_{1}$ cannot separate 0 and $z_{2}-z_{1}$ and we argue as before.

There remains the possibility that $A$ does not separate the points of $G$. But then any unseparated pair differ by a common period of the elements of $A$; if $A \subset C_{0}(G)$ the set $P$ of periods forms a compact subgroup of $G$ and $A$ can be viewed as a subalgebra of $C_{0}(G / P)$ which separates. If instead $A$ lies in $C(G)$ then compactness of $P$ is not needed; $A$ is a subalgebra of $C(G / P)$ which is evidently strictly closed, separating, invariant and proper.

2.1. One can exploit analyticity by excluding its consequences at boundaries. For example for $f \in C_{0}(G)$ suppose that for each nontrivial (continuous but not necessarily open) representation $\sigma: \mathbf{R} \rightarrow G$ there is a coset of the algebraic subgroup $\sigma(\mathbf{R})$ of $G$ which meets $f^{-1}(c)$ in a set of positive finite linear measure, $c=c_{\sigma}$. Then the appropriate translate of $f$ cannot have $f \circ \sigma$ in $H^{\infty}$, and thus the subalgebra of $C_{0}(G)$ generated by $f$ and all its translates is $C_{0}(G / P), P$ again the compact group of periods of $f$. More generally we can replace $c_{\sigma}$ by a set $E \subset \mathbf{C}$ of logarithmic capacity zero by a theorem of Frostman [F, p. 97; Ts, VIII.44]. Thus

Corollary 2. Suppose $f \in C_{0}(G)$ has (*) for each representation $\sigma: \mathbf{R} \rightarrow G$ a translate $f_{x}$ for which $\sigma^{-1}\left(f_{x}^{-1}\left(E_{\sigma}\right)\right)$ has positive finite linear measure for some set $E_{\sigma} \subset \mathbf{C}$ of zero capacity. Then $f$ and its translates generate $C_{0}(G / P)$ as an algebra. 
(In all the remaining examples $P$ will be trivial.)

A simple condition insuring (*) when $G=\mathbf{R}^{n}$ is that $f^{-1}(E)$ have positive Lebesgue measure in $\mathbf{R}^{n}$, for $E$ of zero capacity with $0 \notin E$. For now each $\sigma$ is an isomorphism and by Fubini some coset of any line $\sigma(\mathbf{R})$ in $\mathbf{R}^{n}$ meets $f^{-1}(E)$ in a set of positive measure, necessarily finite since $0 \notin E$. Thus we have

Corollary 3. Let $E \subset \mathbf{C}$ have capacity zero, $0 \notin E$. Suppose $f \in C_{0}\left(\mathbf{R}^{n}\right)$ has $^{6}$ $f^{-1}(E)$ of positive measure. Then $f$ and its translates generate $C_{0}\left(\mathbf{R}^{n}\right)$ as an algebra.

When $\sigma$ must be an isomorphism, as when $G=\mathbf{R}^{n}$, the fact that $a \circ \sigma \in C_{0}(\mathbf{R})$ implies its analytic extension is continuous on $\mathcal{H}_{+} \cup\{\infty\}$, as noted earlier; alternatively $a \circ \sigma$ can be viewed as pulling back into the disc algebra via the standard map of $D$ onto $\mathcal{H}_{+} \cdot$. Consequently we can exploit the argument principle.

COROllary 4. Suppose $G$ has no nontrivial compact subgroups, $0 \neq f \in C_{0}(G)$, and for each nonzero homomorphism $\sigma: \mathbf{R} \rightarrow G$

(a) there are $x_{1}, x_{2}$ in $G$ and $z$ in $\mathbf{C}$ for which the winding numbers $\operatorname{Ind}\left(f_{x_{i}} \circ \sigma, z\right)$ exist and are of opposite sign.

Then $f$ and its translates generate $C_{0}$ as an algebra.

Alternatively in (a) one can assume there is one translate and two $z$ 's, using the fact that $\operatorname{Ind}(h, z)$ is of one sign for all $z$ for which it exists for $h$ or $\bar{h}$ in $A(D)$. There are of course a variety of replacements for (a). For example if $d \lambda=2 d t / \pi\left(1+t^{2}\right)$, which represents $i$ on $H^{\infty}\left(\mathcal{H}_{+}\right)$,

$\left(\mathrm{a}^{\prime}\right)$ there are $x \in G$ and $z \in \mathbf{C}$ for which $\int\left|\log f_{x} \circ \sigma-z\right| d \lambda=-\infty$.

Again just the range of $f_{x} \circ \sigma$ can be wrong, as in

$\left(\mathrm{a}^{\prime \prime}\right)$ there is an $x$ in $G$ for which no bounded connected open set $U$ in $\mathbf{C}$ has $\partial U \subset f_{x} \circ \sigma(\mathbf{R} \cup\{\infty\}) \subset U^{-}$.

Finally, assuming $f$ is smooth enough to yield $f_{x} \circ \sigma(\mathbf{R})$ nowhere dense, one could assume

$\left(\mathrm{a}^{\prime \prime \prime}\right)$ there is an $x$ in $G$ and self-homeomorphism $\tau$ of $\mathbf{R}$ for which $f_{x} \circ \sigma \circ \tau$ is even and 0 at 0 .

Here no point $z \in \mathbf{C} \backslash f_{x} \circ \sigma(\mathbf{R} \cup\{\infty\})$ can lie in the range of an analytic extension since for all such $z, \operatorname{Ind}\left(f_{x} \circ \sigma, z\right)=\operatorname{Ind}\left(f_{x} \circ \sigma \circ \tau, z\right)=0$. Needless to say we could vary these variants of (a) with our $\sigma$.

The Phragmén-Lindelöf result used in Corollary 4 in fact extends to show no bounded analytic function on $D$, continuous on $D^{-} \backslash\{1\}$, can have distinct one sided limits on $\partial D$ at 1 [Ti, 5.64]. Consequently one can form a strict density analogue of the above sort of condition.

CoRollaRy 5. Suppose $f \in C\left(\mathbf{R}^{n}\right)$ has, for each nonzero homomorphism $\boldsymbol{\sigma}: \mathbf{R} \rightarrow \mathbf{R}^{n}$, a translate $f_{x}$ for which $f_{x} \circ \sigma$ has distinct limits at $\pm \infty$. Then the algebra $A$ generated by $f$ and its translates is strictly dense in $C\left(\mathbf{R}^{n}\right)$.

\footnotetext{
${ }^{6}$ For $f$ smooth we could equally well assume some partial $g$ of $f$ is bounded and continuous and has $g^{-1}(E)$ of positive measure. (For by induction such partials are bounded pointwise limits of difference quotients of elements of $A^{-}$.)
} 
Evidently such an $f$ has no periods, so $A$ separates $\mathbf{R}^{n}$. Finally, assuming $f \in C\left(\mathbf{R}^{n}\right)$ has no periods one has exactly the strict analogues of Corollaries 2 and 3 ; and supposing equal limits at $\pm \infty$ for $f_{x} \circ \sigma$, an analogue of Corollary 4 as well.

2.2. For $G=\mathbf{R}^{n}$ and $A$ separating, at least when the semigroup (formed from the strict closure $A^{-}$in $C$ )

$$
\mathcal{S}=\Gamma \cap A^{-}=\mathbf{R}^{n \wedge} \cap A^{-}
$$

is nice enough the entire spectrum of $A$ is the union of analytic discs (of the type described in Corollary 1) and $\mathbf{R}^{n}$; for example if $\mathcal{S}$ is a set of synthesis with dense interior.

Indeed, suppose $\varphi \in M_{A}$, so $\varphi$ is represented by a probability measure $\lambda$, and thus has a strictly continuous extension to $A^{-}$given by $\lambda$. Then $\tau: \gamma \rightarrow \lambda(\gamma)$ is multiplicative on $\delta$, continuous, and so a semicharacter of $\mathcal{\delta}$. Moreover $\tau$ never vanishes. For firstly, if $\tau \equiv 0$ then $\varphi=0$ since $\delta$ is of synthesis. So the relatively open set $S \backslash \tau^{-1}(0) \neq \varnothing$ and thus $\mathcal{S}^{0} \backslash \tau^{-1}(0)$ is a nonvoid open set in $\Gamma=\mathbf{R}^{n}$; let $\gamma_{0}+B \subset$ $\delta^{0} \backslash \tau^{-1}(0)$, where $B$ is a small ball centered at 0 . Now suppose $\gamma \in \tau^{-1}(0)$. Then because $\tau^{-1}(0)$ and $\varsigma^{0} \backslash \tau^{-1}(0)$ are both semigroups and $\tau^{-1}(0)$ an ideal, for $k \in \mathbf{Z}_{+}$

$$
\gamma+k\left(\gamma_{0}+B\right) \subset \tau^{-1}(0) \text { and } k\left(\gamma_{0}+B\right) \subset \delta^{0} \backslash \tau^{-1}(0)
$$

and therefore $\left(\gamma+k \gamma_{0}+k B\right) \cap\left(k \gamma_{0}+k B\right)=\varnothing$ so $\gamma \notin k B-k B=2 k B$ for all $k$. So $\tau^{-1}(0)=\varnothing$ as asserted.

As a consequence $\tau$ has an extension $\bar{\tau}$ to all of the group $\delta-\delta$ (which coincides with $\Gamma$ since $\delta-\delta$ is an open dense subgroup); setting $\bar{\tau}\left(\gamma_{1}-\gamma_{2}\right)=\tau\left(\gamma_{1}\right) / \tau\left(\gamma_{2}\right)$, we have a well-defined homomorphism of $\Gamma$ into $(\mathbf{C} \backslash(0), \cdot)$ and if again $\gamma_{0}+B \subset \varsigma^{0}$ then for $\gamma=\gamma_{1}-\gamma_{2} \in \mathcal{S}-\delta$, on $\gamma+B=\gamma_{1}-\gamma_{2}+B=\gamma_{1}+\gamma_{0}+B-\left(\gamma_{0}+\gamma_{2}\right)$,

$$
\bar{\tau}(\gamma+b)=\tau\left(\gamma_{1}+\gamma_{0}+b\right) / \tau\left(\gamma_{0}+\gamma_{2}\right)
$$

and so is continuous since $\gamma_{1}+\gamma_{0}+B \subset \delta$.

Now we can write $\tau=\tau_{1} \tau_{2}$ with $\tau_{i}$ continuous and multiplicative, $\left|\tau_{1}\right| \equiv 1$ and $\tau_{2}>0$. So $\tau_{1}(\gamma)=\langle x, \gamma\rangle$ for some $x \in \mathbf{R}^{n}=G$, and, since $\Gamma$ is simply connected, $\tau_{2}(\gamma)=e^{-\rho(\gamma)}$, where $\rho$ is a homomorphism into the additive reals, $\geqslant 0$ on $\delta$ since $|\tau(\gamma)| \leqslant 1$ for $\gamma \in \mathcal{S}$. If $\rho$ is trivial, so $\lambda(\gamma)=\tau(\gamma)=\tau_{1}(\gamma)=\langle x, \gamma\rangle=\delta_{x}(\gamma)$, then $\delta_{x}$ and $\lambda$ represent the same strictly continuous functional on the strictly closed span of $\mathcal{S}$. Since $\delta$ is a set of synthesis that set coincides with $A^{-}$, and $\varphi$ is evaluation at $x$. On the other hand if $\rho$ is nontrivial, hence positive somewhere on $\mathcal{S}$, and $\lambda_{z}$ is the Cauchy measure representing $z \in \mathcal{H}_{+}$on $H^{\infty}\left(\mathcal{H}_{+}\right)$then since $\frac{2}{\pi} \int \bar{e}^{i t x} /\left(1+t^{2}\right) d t=$ $\lambda_{i}\left(e^{-i x \cdot}\right)=e^{-|x|}$,

$$
\hat{\rho}^{*} \lambda_{i}(\gamma)=\lambda_{i}(\gamma \circ \hat{\rho})=\lambda_{i}(\rho(\gamma))=e^{-\rho(\gamma)}, \quad \gamma \in \mathcal{S},
$$

so $\delta_{x} * \hat{\rho}^{*} \lambda_{i}(\gamma)=\tau(\gamma)=\lambda(\gamma)$, and thus $\delta_{x} * \hat{\rho}^{*} \lambda_{i}$ represents $\varphi$ on $A$, again by synthesis. Now $z \rightarrow \delta_{x} * \hat{\rho}^{*} \lambda_{z}$ provides our analytic disc through $\varphi$ in $M_{A}$; for the measure, being multiplicative on span $\mathcal{S}$, must be multiplicative on $A^{-}$, while $f: z \rightarrow \delta_{x} * \hat{\rho}^{*} \lambda_{z}(a)$ is analytic for $a \in A$ since sp $f$ lies in $\mathbf{R}_{+}$exactly as in the last step in the proof of Theorem 1 .

The above shows that, under our special hypotheses not only that any $\varphi \in M_{A}$ gives rise to a semicharacter of $\mathcal{S}$ but any continuous semicharacter $\tau$ of $\mathcal{S}$, which 
trivially provides a multiplicative linear functional on span $\mathcal{S}$, is represented by a probability measure, and thus extends to a multiplicative linear functional on (span $\delta)^{-}=A^{-}$; hence $M_{A}$ can be identified with the set $\delta$ of semicharacters of $\delta$. (The same applies if $A$ is a strictly closed subalgebra of $C\left(\mathbf{R}^{n}\right)$ and we interpret $M_{A}$ to be the set of strictly continuous multiplicative linear functionals.) Clearly, in this case, Theorem 1 adds only the fact that $M_{A} \backslash \mathbf{R}^{n} \neq 0$.

3. For a subalgebra $A$ of $A(G)$ or $B(G)$ translation invariance amounts to invariance of the corresponding subalgebra $A_{1}$ of $L^{1}(\Gamma)$ or $M(\Gamma)$ under multiplication by characters, or trigonometric polynomials. By a familiar argument the last implies (and is trivially implied by) the fact that $A_{1}$ is an $L$-subalgebra; $\mu \in A_{1}$ and $\nu \ll|\mu|$ imply $\nu \in A_{1}$. As a consequence we easily obtain the analogue of Theorem 1 for $A \subset A(G)$.

THEOREM 3. If $A \subset A(G)$ is a closed proper translation invariant subalgebra either $A=\left(L^{1}\left(\Gamma_{0}\right)\right)$ for an open subgroup $\Gamma_{0}$ of $\Gamma$, or the conclusions of Theorem 1 apply, and $M_{A}$ contains analytic discs.

Again our analytic discs are bounded by homomorphs of $\mathbf{R}$ in $G$ or a quotient of $G$; thus Corollaries 2 through 5 apply equally well with $A(G)$ in place of $C_{0}(G)$. (For example, here Corollary 3 asserts that if $f \in L^{1}\left(\mathbf{R}^{n}\right)$ has $\hat{f}^{-1}(1)$ of positive measure then the closed subalgebra of $L^{1}\left(\mathbf{R}^{n}\right)$ generated by $\left\{e^{i(t, \cdot)} f: t \in \mathbf{R}^{n}\right\}$, or by $\left\{\chi_{U} f: U\right.$ Borel in $\mathbf{R}^{n}$, is all of $L^{1}\left(\mathbf{R}^{n}\right)$.)

In this context separation by $A$ can be achieved by replacing $G$ by $G / P$ for the (again necessarily compact) group of periods $P$ of $A$; of course in the theorem $\Gamma_{0}=P^{\perp}$ and in our corollaries we obtain $\Gamma=\Gamma_{0}$ again because $P$ must be trivial when $G=\mathbf{R}^{n}$.

For the proof let $S_{\mu}$ denote the closed support in $\Gamma$ of $\mu$ in $A_{1}$, and $S$ the closure of the union of all such $S_{\mu}, \mu \geqslant 0$. Because $S_{\mu * \nu}=\left(S_{\mu}+S_{\nu}\right)^{-}$for two nonnegative measures, $S$ is a semigroup; since $S$ contains a set of positive Haar measure it has interior and generates an open subgroup $\Gamma_{0}$ of $\Gamma$. For simplicity we can take $\hat{A_{1}}=\hat{A}$ as subalgebra of $A\left(G / \Gamma_{0}^{\perp}\right)$; replacing $G$ and $\Gamma$ by $G / \Gamma_{0}^{\perp}$ and $\Gamma_{0}$, we can thus view $A$ as also separating $G$, and $S$ as generating $\Gamma$.

Suppose $S \neq \Gamma$. By Theorem 2 we have a (continuous) homomorphism $\rho_{1}$ of $\Gamma$ onto $\Pi$, an ordered group which is either $\mathbf{R}$ or discrete, $\geqslant 0$ on $S$, and nontrivial on $S$ since $S$ generates $\Gamma$. In case $\Pi$ is discrete we can follow $\rho_{1}$ by a nonnegative homomorphism into $\mathbf{R}$ of an open subgroup, which composes with $\rho_{1}$ to yield a homomorphism $\rho$ of an open subgroup $\Gamma_{1}$ of $\Gamma$ into $\mathbf{R}$, with $\rho\left(S \cap \Gamma_{1}\right) \subset \mathbf{R}_{+}$, $\neq\{0\}$ : we have only to select $s_{0} \in S$ with $\pi_{0}=\rho_{1}\left(s_{0}\right)>0$, note that the subgroup of $\Pi$ of elements bounded by $\pi_{0}$ (as in (5)), modulo its subgroup of elements infinitely small relative to $\pi_{0}$, is archimedian, and thus order isomorphic to a subgroup of $\mathbf{R}$.

Hence if $S$ is proper we have a continuous homomorphism $\rho$ of an open subgroup $\Gamma_{1}$ of $\Gamma$ for which $\rho\left(S \cap \Gamma_{1}\right) \geqslant 0, \neq\{0\}$, while $S \backslash \Gamma_{1}$ is an ideal in $S$. Since $\operatorname{sp} \hat{\mu} \subset S_{\mu}$ we see $(m * \hat{\mu}) \circ \hat{\rho}$ extends to be analytic on a half-plane or disc as before via $1.5,1.7$ and the proof of Corollary 1 where $m$ is Haar measure of $\Gamma_{1}^{\perp}$. (Alternatively from 
Theorem 2 we could produce a positive semicharacter $\chi$ of $S$, zero on $\Gamma_{1} \backslash S$, and follow Taylor by setting

$$
\varphi_{z}(\mu)=\int \chi^{z} d \mu, \quad \operatorname{Re} z \geqslant 0
$$

since $\chi=e^{-\rho}$ on $\Gamma_{1}$ for $\rho$ as above we obtain $\varphi_{i y}(\mu)=\int_{\Gamma_{1}} e^{-i y \rho(\gamma)} \mu(d \gamma)=$ $\left.\int_{\Gamma_{1}}\langle\hat{\rho}(y), \gamma\rangle \mu(d \gamma)=\left(\mu_{\Gamma_{1}}\right) \hat{(\rho}(y)\right)=(m * \hat{\mu}) \circ \hat{\rho}(y)$, showing we would obtain the same analytic disc.)

There remains the possibility that $S=\Gamma$. Then because $A_{1}$ is an $L$-algebra in any neighborhood $N_{\gamma}$ of $\gamma$ we can find the support of a nonzero element of $A_{1}$, which we can take to correspond to a nonnegative bounded $L^{1}$ function $f$; choosing $f_{1}$ supported by $N_{\gamma}$ and $f_{2}$ supported by $N_{1}$ we have the open support of the continuous function $f_{1} * f_{2} \subset N_{1}+N_{\gamma}$. Thus $S$ contains a dense open subset $U$, the union of the open supports of the $\mu \geqslant 0$ in $A_{1}$ corresponding to continuous nonnegative elements of $L^{1}$ in $A_{1}$. Since $U$ is trivially a semigroup, and dense, it must be $G: V=(-U) \cap$ $U \neq \varnothing$, so $U$ contains the neighborhood $W=V+V=V-V$ of 1 , whence $(\gamma+W) \cap U \neq \varnothing$ implies $\gamma \in U$.

Now since evidently $\chi_{U} \cdot L^{1} \subset A_{1}$ follows from the definition of $U$ and the fact that $A_{1}$ is an $L$-algebra, we are done.

In the case of an invariant subalgebra of $B(G)$ and the corresponding $L$-subalgebra of $A$ of $M(\Gamma)$ the situation is more complicated, and analytic discs may exist and lie far from our group $G$; thus only special forms of our corollaries obtain. We now rely heavily on the work of Taylor, and it is only because of that work that we can almost precisely describe the settings in which analytic discs arise (though not in a completely transparent manner). The sole gap that remains would be closed by a negative answer to a question left open by Taylor [T2, p. 112].

Because a generalized character of $A$ whose modulus is nonidempotent immediately leads to an analytic disc in the spectrum, if such discs fail to exist we can locate $A$ in the sum of two subalgebras of $M(\Gamma)$ described by Taylor in [T2]. Let $\mathcal{T}$ denote the set of all locally compact group topologies $\tau$ for $\Gamma$ at least as fine as the original, with $\Gamma_{\tau}$ the corresponding 1.c.a. group. The radical of $L^{1}\left(\Gamma_{\tau}\right), L^{1}\left(\Gamma_{\tau}\right)^{1 / 2}$, is the intersection of all maximal ideals of $M\left(\Gamma_{\tau}\right)$ containing $L^{1}\left(\Gamma_{\tau}\right)$. We view all such measures as lying in $M(\Gamma)$, and set $M_{1}(\Gamma)=\Sigma_{\tau} L^{1}\left(\Gamma_{\tau}\right)^{1 / 2}$, which is the first subalgebra. (The sum is in fact a direct sum of mutually singular subspaces.) The second of Taylor's subalgebras $M_{2}(\Gamma)$ is more recondite; it consists of those measures which, carried to the structure semigroup, are supported by the union of all maximal subgroups (as are those in $M_{1}(\Gamma)$ ) but which vanish on each such subgroup; whether $M_{2}(\Gamma)$ can be nontrivial is the question left open by Taylor. Excluding generalized characters of $A$ with nonidempotent moduli implies, by [T2, 2.3] applied to $M=$ $M(\Gamma)$, that

$$
A \subset M_{1}(\Gamma)+M_{2}(\Gamma)
$$

what one can conclude when no analytic discs exist is that if $A_{\tau}=A \cap L^{1}(\Gamma)^{1 / 2}$, then for each $\tau$ in $\sigma$ either:

(a) $A_{\tau}=0$, 
(b) $L^{1}\left(\Gamma_{\tau}^{*}\right) \subset A_{\tau} \subset L^{1}\left(\Gamma_{\tau}^{*}\right)^{1 / 2}$, where $\Gamma_{\tau}^{*}$ is an open subgroup of $\Gamma_{\tau}$, or

(c) $A_{\tau} \cap L^{1}\left(\Gamma_{\tau}\right)=0$, and the support semigroup of $A_{\tau}$ is a group.

(Here the support semigroup of an $L$-algebra is simply the closure of the union of the closed supports of its elements, necessarily a semigroup.) In all of these cases the support semigroup of $A_{\tau}$ is a group; conversely that condition implies (a), (b) or (c) holds and, at least when $A \cap M_{2}(\Gamma)=0$, that no analytic discs exist.

Theorem 4. Let $A$ be an L-subalgebra of $M(\Gamma)$. If the spectrum of $A$ contains no analytic discs then $A$ is the sum of the two mutually singular L-subalgebras $A \cap M_{1}(\Gamma)$, $A \cap M_{2}(\Gamma)$, where each component $A_{\tau}=A \cap L^{1}\left(\Gamma_{\tau}\right)^{1 / 2}$ for $\tau \in \mathcal{T}$ has its support semigroup (void or) a group, or satisfies (a), (b) or (c).

Conversely these conditions imply the elements of $A \cap M_{1}(\Gamma)$ are constant on any analytic disc in the spectrum, so all such discs necessarily arise in the spectrum of the ideal $A \cap M_{2}(\Gamma)$ of $A$.

Suppose we have no analytic discs in the spectrum so (11) holds. Recall that for each $\tau$ in $\mathcal{T}$ and each $\mu$ in $M(\Gamma)$ we can uniquely write $\mu=\mu_{\tau}+\mu_{\tau}^{\prime}$ where $\mu_{\tau}$ is carried by a $\tau \sigma$-compact set and $\mu_{\tau}^{\prime}$ vanishes on all $\tau$ compacta, while $\mu \rightarrow \mu_{\tau}$ is multiplicative; thus viewing $M\left(\Gamma_{\tau}\right)$ as a subset of $M(\Gamma)$ we can imbed the spectrum of $A \cap M\left(\Gamma_{\tau}\right)$ into that of $A \cap M(\Gamma)$. Morover, since $L^{1}\left(\Gamma_{\tau}\right)^{1 / 2}$ is an ideal of $M\left(\Gamma_{\tau}\right)$ the spectrum of $A_{\tau}=A \cap L\left(\Gamma_{\tau}^{1}\right)^{1 / 2}$ imbeds in that of $A \cap M\left(\Gamma_{\tau}\right)$, hence in that of $M(\Gamma)$. So for each $\tau$ the spectrum of $A_{\tau}$ contains no analytic disc.

Because of Theorem 3 we can assert $A_{\tau} \cap L^{1}\left(\Gamma_{\tau}\right)=0$ or $A_{\tau} \cap L^{1}\left(\Gamma_{\tau}\right)=L^{1}\left(\Gamma_{\tau}^{*}\right)$ for an open subgroup $\Gamma_{\tau}^{*}$ of $\Gamma_{\tau}$; for if both fail then the spectrum of $A_{\tau} \cap L^{1}\left(\Gamma_{\tau}\right)$ contains analytic discs by Theorem 3 , and since this algebra is an ideal in $A_{\tau}$, so must the spectrum of $A_{\tau}$. When the second alternative holds $\Gamma_{\tau}^{*}$ must be the support semigroup of $A_{\tau}$ since otherwise we have elements of the ideal $A_{\tau} \cap L^{1}\left(\Gamma_{\tau}\right)$ not supported by $\Gamma_{\tau}^{*}$; and because $L^{1}\left(\Gamma_{\tau}^{*}\right)^{1 / 2}=L^{1}\left(\Gamma_{\tau}\right)^{1 / 2} \cap M\left(\Gamma_{\tau}^{*}\right)$ holds ${ }^{7}$ for any open subgroup $\Gamma_{\tau}^{*}$, the right-hand inclusion in (b) follows, and (b) holds.

Now if (a), (b) and (c) fail then $A_{\tau} \cap L^{1}\left(\Gamma_{\tau}\right)=0$ but $A_{\tau}=A \cap L^{1}\left(\Gamma_{\tau}\right)^{1 / 2} \neq 0$ and the support semigroup $S$ of $A$ is not a group. So exactly as in the proof of Theorem 3 we can produce a positive semicharacter $\chi$ of $S$ with values in $(0,1)$ on an open subset of $S$, and

$$
\varphi_{z}(a)=\int \chi^{z} d a, \quad \operatorname{Re} z \geqslant 0,
$$

provides an analytic disc.

Conversely, suppose the support semigroup of $A_{\tau}$ is always void or a group $\Gamma_{\tau}^{0}$. If $A_{\tau} \cap L^{1}\left(\Gamma_{\tau}\right) \neq 0$ then $\Gamma_{\tau}^{0}$ must be open, and by just the argument of Theorem 3,

7 Trivially, $L^{1}\left(\Gamma_{\tau}^{*}\right)^{1 / 2} \subset L^{1}\left(\Gamma_{\tau}\right)^{1 / 2} \cap M\left(\Gamma_{\tau}^{*}\right)$ since any multiplicative linear functional on $M\left(\Gamma_{\tau}\right)$ vanishing on $L^{1}\left(\Gamma_{\tau}\right)$ restricts to $M\left(\Gamma_{\tau}^{*}\right)$ to vanish on $L^{1}\left(\Gamma_{\tau}^{*}\right)$, hence vanishes on $L^{1}\left(\Gamma_{\tau}^{*}\right)^{1 / 2}$. For the reverse, it suffices to see each element $\varphi$ of the spectrum of $M\left(\Gamma_{\tau}^{*}\right)$ vanishing on $L^{1}\left(\Gamma_{\tau}^{*}\right)$ is such a restriction. But $\left\{\mu \in M\left(\Gamma_{\tau}\right):\left.\mu\right|_{\gamma \Gamma_{\tau}^{*}} \in \delta_{\gamma} * \varphi^{-1}(0), \gamma \in \Gamma_{\tau}\right\}$, the set of measures coinciding with translates of $\varphi^{-1}(0)$ on translates of $\Gamma_{\tau}^{*}$, is a closed proper ideal containing $L^{1}\left(\Gamma_{\tau}\right)$, and so lies in the kernel of a $\psi$ in the spectrum of $M\left(\Gamma_{\tau}\right)$. Clearly $\psi$ annihilates $L^{1}\left(\Gamma_{\tau}\right)$, and since $\psi^{-1}(0) \supset \varphi^{-1}(0)$ and cannot contain $M\left(\Gamma_{\tau}^{*}\right)$ unless it contains all translates $\delta_{\gamma} * M\left(\Gamma_{\tau}^{*}\right)$, hence all $M\left(\Gamma_{\tau}\right), \psi \mid M\left(\Gamma_{\tau}^{*}\right)=\varphi$. 
$A_{\tau} \cap L\left(\Gamma_{\tau}\right)=L^{1}\left(\Gamma_{\tau}^{0}\right)$, so (b) follows for $\Gamma_{\tau}^{0}=\Gamma_{\tau}^{*}$ as before. On the other hand, if $A_{\tau} \cap L^{1}\left(\Gamma_{\tau}\right)=0$ clearly (a) or (c) holds.

Now Taylor [T3, p. 84] calls $A$ balanced if $A \cap L^{1}\left(\Gamma_{\tau}\right)$ is always 0 or $L^{1}\left(\Gamma_{\tau}^{*}\right)$ for a (necessarily open) subgroup $\Gamma_{\tau}^{*}, \tau \in \mathcal{T}$; thus our alternatives (a)-(c) imply $A$ is balanced. So by [T3, 9.1.2] for $a$ in $A_{\tau}$ its spectrum in $A_{\tau}$ coincides with its spectrum in $M(\Gamma)$, or $\operatorname{sp}_{A_{\tau}} a=\operatorname{sp}_{M(\Gamma)} a$. Because of our homomorphism $\mu \rightarrow \mu_{\tau}$ the latter coincides with $\operatorname{sp}_{M\left(\Gamma_{\tau}\right)} a$, which is precisely $\hat{a}\left(\Gamma_{\tau}\right) \cup\{0\}$, essentially by the definition of $L^{1}\left(\Gamma_{\tau}\right)^{1 / 2}$. Thus $\operatorname{sp}_{A_{\tau}} a=\hat{a}\left(\Gamma_{\tau}\right) \cup\{0\}$, and the image of $\Gamma_{\tau}^{\hat{\imath}}$ in the spectrum of $A_{\tau}$ provides a boundary; indeed since $A_{\tau}$ is an $L$-algebra, $\hat{A_{\tau} \hat{\imath}}$ on $\hat{\Gamma_{\tau}}$ is translation invariant, so the image is that of the quotient group $\Gamma_{\tau}^{\wedge} / \Gamma_{\tau}^{* \perp}$, which, again by invariance, must yield the Srlov boundary.

Suppose then that some element $a_{0}$ of $A_{\tau}$ has its Gelfand transform $\tilde{a}_{0}$ nonconstant on our analytic disc $z \rightarrow \varphi_{z}$. In case (b) each $\tilde{a}$ in $L^{1}\left(\Gamma_{\tau}^{*}\right)$ is constant on our disc since those with $\hat{a}$ real valued $\operatorname{span} L^{1}\left(\Gamma_{\tau}^{*}\right) \subset A_{\tau}$, and these yield analytic functions with range $\operatorname{sp}_{A_{\tau}} a=\hat{a}\left(\Gamma_{\tau}\right) \cup\{0\} \subset \mathbf{R}$, and so are constant. Thus $a_{0}$ must lie outside $L^{1}\left(\Gamma_{\tau}^{*}\right)$. But if $a \in L^{1}\left(\Gamma_{\tau}^{*}\right),\left(a * a_{0}\right) \tilde{c}$ constant on our disc implies $\tilde{a}=0$ on our disc, so $\varphi_{z}$ vanishes on $L^{1}\left(\Gamma_{\tau}^{*}\right)$, and by definition it vanishes on all $L^{1}\left(\Gamma_{\tau}^{*}\right)^{1 / 2}$, so case (b) cannot arise, and we must have case (c).

Now since the support semigroup of $A_{\tau}$ is a group $\Gamma_{\tau}^{*}$ we can construct an element $a_{1} \geqslant 0$ whose support $\Gamma_{1}$ is itself an open subgroup of $\Gamma_{\tau}^{*}$ while $a_{0} \ll a_{1}$. Indeed replacing $a_{1}$ by a power series in $a_{1}$ we can assume $a_{1} * a_{1} \ll a_{1}$.

Consequently, from [T3, 9.2.2] each $\varphi_{z}$ in our disc is given on $L^{1}\left(a_{1}\right)$ by $a \rightarrow \int f d a$, $f \in L^{\infty}\left(a_{1}\right)$, with $f\left(\gamma \gamma^{\prime}\right)=f(\gamma) f\left(\gamma^{\prime}\right)$ a.e. $a_{1} \times a_{1}$. But because $\Gamma_{\tau}^{\wedge} / \Gamma_{\tau}^{* \perp}$ provides the Silov boundary, $\varphi_{z}$ is represented by a probability measure $\nu_{z} \in M\left(\Gamma_{\tau}^{\wedge} / \Gamma_{\tau}^{* \perp}\right)$ :

$$
\varphi_{z}(a)=\int \hat{a} d \nu_{z}=\int \hat{\nu}_{z} d a, \quad a \in A_{\tau} .
$$

Thus $\hat{\nu}_{z}\left(\gamma \gamma^{\prime}\right)=\hat{\nu}_{z}(\gamma) \hat{\nu}_{z}\left(\gamma^{\prime}\right)$ a.e. $a_{1} \times a_{1}$, so for $\gamma \notin N$ this holds for $\gamma^{\prime} \notin N_{\gamma}$ where $N$ and $N_{\gamma}$ are $a_{1}$-null. Since $N$ and $N_{\gamma}$ are necessarily nowhere dense in $\Gamma_{1}$ and $\hat{\nu}_{z}$ is continuous we conclude $\hat{\nu}_{z}$ is multiplicative on all $\Gamma_{1}$. Finally since $\Gamma_{1}$ can be any (large) $\sigma$-compact open subgroup of $\Gamma_{\tau}^{*}, \hat{\nu}_{z}$ is multiplicative on all of $\Gamma_{\tau}^{*}$, hence is a character. Thus $\nu_{z}$ must be a point mass on the dual group $\Gamma_{\tau}^{\hat{}} / \Gamma_{\tau}^{* \perp}$, and so our disc lies entirely in the Šlov boundary.

Now the identity of $\Gamma_{\tau}^{\wedge} / \Gamma_{\tau}^{* \perp}$ is a generalized peak point for $\hat{A_{\tau}}$ since for our $a_{1}$, $\hat{a}_{1}(1)=\left\|\hat{a}_{1}\right\|_{\infty}=\left\|a_{1}\right\|$ while $\hat{a}_{1}(x)=\left\|a_{1}\right\|$ implies $x=1$ on the support of $a_{1}$, an arbitrary (large) $\sigma$-compact open subgroup of $\Gamma_{\tau}^{*}$. By translation invariance each point of $\Gamma_{\tau}^{\hat{\lambda}} / \Gamma_{\tau}^{* \perp}$ is a generalized peak point; since no point of an analytic disc can be one we have our contradiction, completing our proof.

Note that when no analytic discs exist we can also conclude the support semigroup of $A \cap M_{2}(\Gamma)$ is a group; otherwise we obtain an analytic disc in its spectrum from a positive semicharacter as before, which imbeds in the spectrum of $A$ since $A \cap M_{2}(\Gamma)$ is an ideal. But we cannot obtain a converse, as with $A_{\tau}$, since $\operatorname{sp}_{M(\Gamma)} a$ is no longer easily related to a Fourier-Stieltjes transform.

Finally, we should note that our corollaries, formulated in terms of just the transform algebra $A \subset B(G)$, can really only hold when $A \subset L^{1}(\Gamma)^{1 / 2}$, since a disc 
nonconstant on $A_{\tau}$ has its boundary in a homomorph of $\mathbf{R}$ in a quotient of $\hat{\Gamma_{\tau}}$, which is strictly larger than $G$. And in case $A \subset L^{1}(\Gamma)^{1 / 2}$, our conclusion (if analyticity must fail) is less precise.

REMARK (ADDED IN PROOF DECEMBER 29, 1982). One can regard Theorem 2 as an analogue in the group setting of the existence of support functionals for cones in Banach spaces. In this sense there is an analogue of the separation theorem which follows from the proof of Theorem 1, viz.: if $S$ is a closed generating subsemigroup of an l.c.a. group $\Gamma$ which contains the identity, and $x \in \Gamma$ has $x^{n} \notin S$ for all $n \geqslant 1$, then there is a half of $\Gamma$ containing $S$ but not $x$.

One need only note that (in our proof) before the first real enlargement of $S=S_{1}$ or, more properly of $S_{2}=\pi_{1} S_{1}, y=\pi_{1} x$ can be adjoined to $S_{2}^{-1}$ so as to preserve (4), since $y^{n} s^{-1}=y^{-k} s^{\prime}$ for $s, s^{\prime} \in S_{2}$ and $n, k \geqslant 0$ implies $y^{n+k}=s, s^{\prime} \in S_{2}$, which is excluded by hypothesis. Then the $\rho$ and $\Pi$ of Theorem 2 have $S \subset \rho^{-1} \Pi_{+}$and $x \notin \rho^{-1}\left(\Pi_{+}\right)$.

\section{REFERENCES}

[AS] R. Arens and I. M. Singer, Generalized analytic functions, Trans. Amer. Math. Soc. 81 (1956), 379-393.

[B] J. E. Björk, Compact groups operating on Banach algebras, Math. Ann. 205 (1973), 281-297.

[F] O. Frostman, Potential d' equilibre et capacité..., Thesis, M. Lund Univ. Mat. Sem. 3 (1935), 1-118.

[G] T. W. Gamelin, Uniform algebras, Prentice-Hall, Englewood Cliffs, N.J., 1969.

[Gi] V. M. Gichev, Maximal ideal spaces of invariant algebras, Functional Anal. Appl. 13 (1979), 216-217.

[Gl.1] I. Glicksberg, Convolution semigroups of measures, Pacific J. Math. 9 (1959), 51-67.

[GI.2] __ Some uncomplemented function algebras, Trans. Amer. Math. Soc. 111 (1964), 121-137.

[Gl.3] _ Bishop's generalized Stone-Weierstrass theorem for the strict topology, Proc. Amer. Math. Soc. 14 (1963), 329-333.

[H] C. S. Herz, The spectral theory of bounded functions, Trans. Amer. Math. Soc. 94 (1960), 181-232.

[HR] E. Hewitt and K. A. Ross, Abstract harmonic analysis. I, Springer-Verlag, New York, 1970.

[N] M. A. Naimark, Normed rings, Noordhoff, Groningen, 1959.

[R] H. Reiter, Classical harmonic analysis and locally compact groups, Oxford Univ. Press, Oxford, 1968.

[Ri] M. A. Rieffel, A characterization of commutative group algebras and measure algebras, Trans. Amer. Math. Soc. 116 (1965), 32-65.

[Ru] W. Rudin, Fourier analysis on groups, Interscience, New York, 1962.

[T1] J. L. Taylor, Convolution measure algebras with group maximal ideal spaces, Trans. Amer. Math. Soc. 128 (1967), 257-263.

[T2] _ L-subalgebras of $M(G)$, Trans. Amer. Math. Soc. 135 (1969), 105-113.

[T3] __, Measure algebras, Regional Conf. Ser. in Math., No. 16, Amer. Math. Soc., Providence, R.I., 1972.

[Ti] E. C. Titchmarsh, The theory of functions, 2nd ed., Oxford Univ. Press, London, 1939.

[Ts] M. Tsuji, Potential theory in modern function theory, Maruzen, Tokyo, 1959.

[W] A. D. Wallace, The structure of topological semigroups, Bull. Amer. Math. Soc. 6 (1955), 95-112.

[We] A. Weil, L'intégration dans les groupes topologiques et ses applications, Actualités Sci. Indust. 869 (1940).

Department of Mathematics, University of Washington, Seattle, Washington 98195. 